\title{
Signs, curls, and time variations: learning to appreciate Faraday's law
}

\author{
Ari Sihvola, Johanna Leppävirta, and Henrik Kettunen \\ Aalto University, School of Electrical Engineering, Department of Radio Science and Engineering, Finland \\ *corresponding author, E-mail: ari.sihvola@aalto.fi
}

\begin{abstract}
In this article, we present the analysis of a study on the development of conceptual understanding of dynamic electromagnetic fields of electrical engineering students in Finland. The focus of the study was teaching and understanding of Faraday's law. A coil with two light-emitting diodes and a strong permanent magnet were used with which the induced electromotive force could be made visible. However, the field and flux directions, temporal changes, and topological constellations within this setting determine in a subtle manner the character of the resulting electric effect. The demonstration was used on electromagnetic field theory classes at Aalto University, Finland, to assess the conceptual understanding of the students. Drawing from the Peer Instruction principle, the students were asked to fill in a questionnaire concerning this experiment, first on their own, and once again after discussing with their neighbors in the classroom. They were asked about the direction of the electric force and the confidence of their answer. The results show that the answer is not very obvious: students tend to vote for the wrong answer. The Peer Instruction discussion greatly improves the situation. Also, the confidence of the students increases thanks to the discussion period with neighbors. The results, however, seem to be somewhat sensitive to the exact constellation and the administration of the experiment.
\end{abstract}

\section{Introduction}

"Of all the phenomena of physics, those associated with Faraday's law are among the most persistently fascinating and puzzling."

It is easy to agree with this summation by Robert $\mathrm{H}$. Romer [1]. In the curriculum of electrical engineering, one of the most challenging topics is the set of Maxwell equations, both for the student and also for the teacher. The divergence relations can certainly be approached from the intuitive understanding of field lines that emanate from a charge. The Gaussian view of the total electric flux through a closed surface being equal to the enclosed net charge leads to Coulomb's law, which again, as an analogy to the forces of Newtonian gravitation, strengthens the conceptual framework of physical understanding in the students' minds. Along with this picture, it is easy to connect the vanishing divergence of the magnetic flux density with the nonexistence of magnetic monopoles: the magnetic field lines have neither sources nor sinks, they always form closed loops.

More challenging is to bring the students to grasp the meaning of the Maxwell curl equations: Ampère's law supplemented with the displacement current term, and Faraday's law. To acquire a mental picture of the "rotor" operation, or curl, of a vector field requires spatial imagination. In addition, when the curl equations also involve temporal differentiations, the resulting combination of symbols and operators is not instantly digestible for a freshman student.

In this article, we focus on Faraday's law and our experiences in teaching to find the most effective ways to help students to develop conceptual understanding of the manner how a varying magnetic field brings forth an electric force.

\section{Faraday's law: differential and macroscopic forms}

In the language of vectors, Faraday's law reads

$$
\nabla \times \vec{E}(\vec{r}, t)=-\frac{\partial \vec{B}(\vec{r}, t)}{\partial t}
$$

where $\vec{E}$ is the electric field vector and $\vec{B}$ is the magnetic flux density, depending on the space $\vec{r}$ and time $t$. This relation is a (partial) differential equation, in other words it is satisfied pointwise: at any point in any time instant, the time variation of the magnetic field dictates the curl of the electric field. Furthermore, the law gives an exact quantitative connection for the relation.

However, the conceptual understanding of this relation requires thinking in not pointwise and differential but rather integrated terms. Use of Stokes's theorem gives the following relation. Given an open surface, the time variation of the total magnetic flux $\Phi$ through it gives the electromotive force over the closed line that forms the boundary of this surface:

$$
\mathrm{emf}=-\frac{\mathrm{d} \Phi}{\mathrm{d} t}
$$

This relation - many students would agree - is considerably more intuitive than the differential form of Faraday's law (1).

However, even if relation (2) is simple in form and straightforward to use in quantitative enumeration of the voltage that is created by changing magnetism, there are still several possibilities to get the direction (the sign) of the electromotive force wrong. For the first, it is the time 

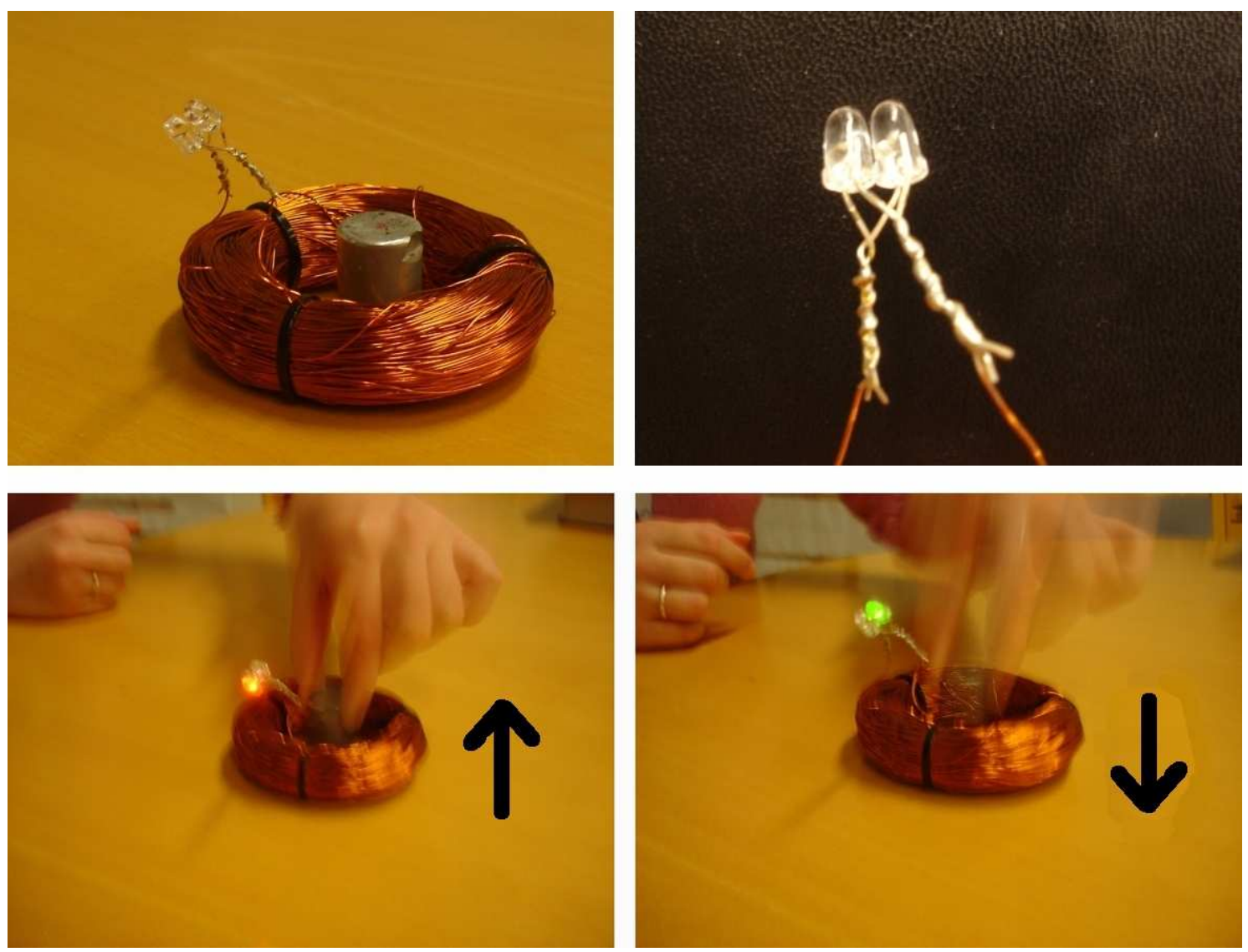

Figure 1: The device (top left panel) consists of copper wire coiled into a large number of turns, with ends soldered to lightemitting diodes (top right panel), one red, one green, with opposite polarity connections. A bar magnet is drawn off or put back into the center of the coil (bottom panels). The direction of the movement and the orientation of the magnet determines which of the LEDs (red or green) flashes.

derivative of the flux rather than the flux itself which matters for the induced force. In addition, the right-hand-rule convention between the flux direction and the direction of the bounding contour has to be correctly accounted for, and thirdly, the additional minus sign does not make it easier to decipher the final direction of the electromotive force. However, Lenz's law gives useful advice in determining the direction of the induced electromotive force.

In electromagnetics textbooks, there are several different approaches to cover Faraday's law. Certain books outright introduce the Maxwell equations in their full glory with all curls and divergences $[2,3,4,5]$. Others reach towards the full-wave electromagnetics in a stepwise manner: first teaching the principles of static fields (electrostatics, steady currents, and magnetostatics), and only then focus on Faraday's law, first in the integral form (Equation (2)), from which, by exploiting Stokes' theorem, arrive in the differential form (1) $[6,7,8,9]$. An even softer path is to move from statics to dynamics through slowlyvarying fields. In connection of such fields, Faraday's law is embraced but not yet the displacement current term in the Ampère-Maxwell law [10, 11, 12].

\section{Simple demonstration of the electromotive effect}

Fortunately, it is rather easy to demonstrate the message carried by Faraday's law. Let us consider a copper wire whose ends are connected by an LED (light-emitting diode) and which is coiled into a large number of turns. There is no battery in the circuit, and hence the LED does not emit light. However, when a bar magnet is moved into the coil or drawn away from the coil, a voltage is excited and the LED emits a light pulse, depending on the direction of the north pole of the magnet and the speed with which the magnet is moved.

In fact, the most effective way is to solder two LED diodes (with different colors) on the wire ends, such that the polarities are opposites (see Figure 1). Then they serve as indicators for a (sufficiently speedy) increase or decrease of the magnetic flux through the coil.

\section{Peer Instruction and Faraday's law demonstration}

The device shown in Figure 1 has been used to demonstrate Faraday's law in electromagnetics courses at the Aalto Uni- 
versity in Finland. To study the students' true understanding of the connections between the magnetic field change and the induced electromotive force, a quantitative study was conducted during the same lecture when Faraday's law was covered on the mathematical and physical contexts. The coil experiment was applied and the students were asked to predict the direction of the induced current $(=$ the color of the LED that would flash) and explain their reasoning. The study was performed in connection with the course "Dynamic Field Theory" in the Department of Electrical and Communications Engineering at the Helsinki University of Technology (presently Aalto University) in 2005 and in 2011. The number of students participating in the study was 78 in 2005 and 99 in 2011. The Finnish-language textbooks that were used in this field theory course and its companion course "Static Field Theory" have been written by the teacher and his colleague $[13,14]$.

In particular, the Peer Instruction (PI) method developed by Mazur [15] was used in order to engage students in active participation. In PI, students first consider the given problem alone. After the first guesses, the students are asked to discuss the problem with their close neighbors in the classroom and then reconsider their responses.

In more detail, the study was administered in the following manner.

\subsection{Faraday' law in the classical manner}

The lecture starts with the introduction of Maxwell equations. Then the focus moves into one of these, the Faraday's law. The teacher presents the law, both in its differential (1) and macroscopic (2) forms, and explains and interprets its physical meaning.

\subsection{Demonstration of the effect}

Next, the teacher puts the coil on the table in front of the classroom and explains that he will start changing the magnetic field by moving the magnet inside the coil and out of it. First, by putting the magnet very gently on the table in the center on the coil, nothing happens, and also taking it away very slowly, no LED will flash. However, taking it quickly enough, the red light flashes (the color of course depends on whether the north pole or south pole is on top-the same movement with the bar magnet upside down would switch the color). And reversely, putting the magnet back fast, the result is a flash of green light (note that this reverse action is more difficult to manage because the magnet easily hits the table surface, and a sufficiently speedy movement is necessary to produce a clear flash).

\subsection{Preparation for the questionnaire}

The teacher repeats the demonstrations a couple of times, always explaining the effect and emphasizing the connection of the speed of the movement to the time derivative in the Faraday's law. Then the teacher says that he would ask the students to respond to the question what would happen if he left the magnet sitting in the center of the coil and would lift the coil up, of course swiftly enough. Would there be LED light? And if yes, what color? The teacher repeats the lifting of the magnet (red) and setting it back (green) once more.

\subsection{Pre-discussion responses}

The students are given a questionnaire where they fill in their prediction what happens (red light / green light / no light) when the coil will be lifted and the magnet remains stationary. In addition, they are supposed to mark their confidence about their answer (whether they are definitely sure or probably sure about it, or just guessing). Furthermore, they are asked to explain their reasoning leading to this answer.

\subsection{Data fusion}

After having filled in their answers, the students are asked to discuss the phenomenon with their neighbors for around five minutes. They are asked to find their way closer to the correct solution by arguing for their own explanations and learning from the others.

\subsection{Post-discussion responses}

After the discussion period, the students give their answers again and also rate their confidence level on this new answer, which, of course, may or may not be the same as their pre-discussion answer and confidence.

\subsection{Mother Nature gives the correct answer}

Finally, the teacher does the experiment and lifts the coil, the magnet remaining on the table. And, nonintuitively, the red light flashes. The students are asked to write down their thoughts about learning the correct answer to the question.

\section{Results}

The data consisted of two sets of filled questionnaires, one from the year 2005 (78 replies) and the other from 2011 (99 replies). The results are collected in Figure 2.

In graphical form, the results are shown as histograms in Figure 3. The height of the columns (red, green, no light) measure the number of student responses, and the shading of the column indicates the confidence. Here both years' (2005 and 2011) responses are collected together.

\subsection{Color of the flash}

From the results, the following observations can be made. Firstly, as is easy to believe, extremely few students expected that there is no flash when the coil is lifted. This is what one also would expect.

However, as to the prediction which is the color which will flash, there is an interesting split between the red light answers (correct) and green light ones. Indeed, when the students replied on their own (pre-discussion answer), the majority of them chose the wrong answer: we can observe 


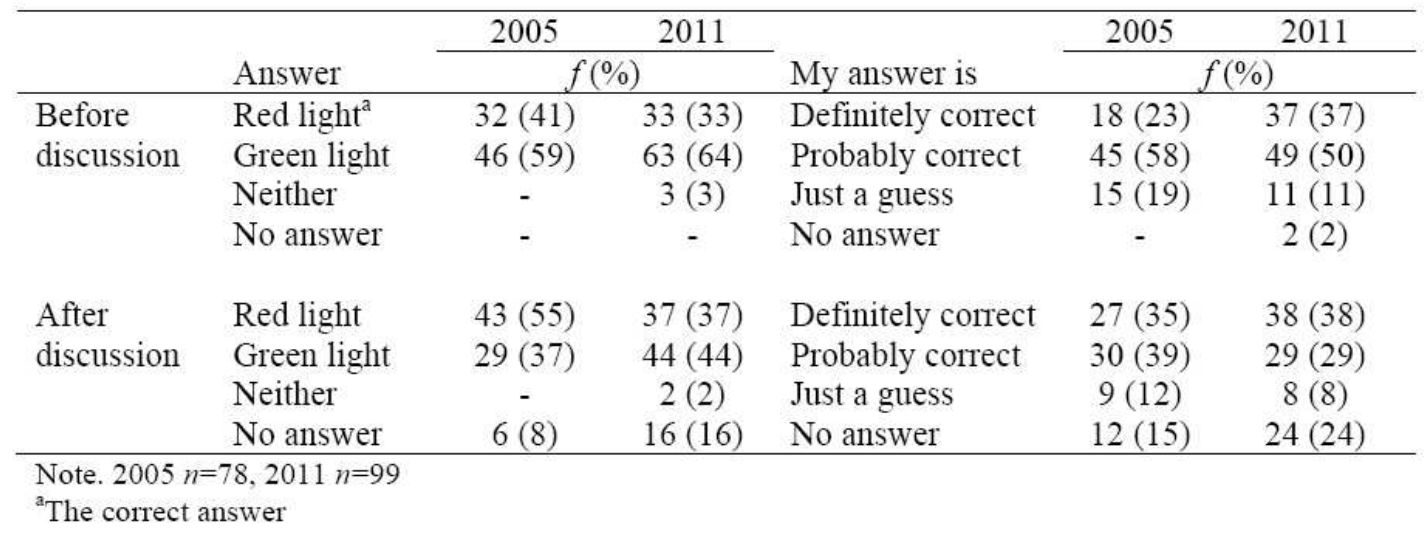

Figure 2: The distribution of students' answers and self-rated confidence levels before and after the discussion.

in Table 1 that there are about twice as many greens as reds.

This tendency towards the green color is in some sense understandable: if one reasons logically from the point of view of relative movement of the magnet and the coil, the following analysis is obvious. Since green light is affiliated with downward movement of the magnet (with stationary coil), then also the upward movement of the coil (with stationary magnet) could be expected to produce green light.

This is also reflected in the students' questionnaires where they verbally justify their responses:

Student A: "When the magnet moves downwards, the green light flashes. Movement is relative, so the same thing happens when the coil moves upwards."

Student B: "I don't think it really matters, which one is moving, the magnet or the coil, as long as the direction is the same."

However, the essence in the emergence of the induced electromotive effect is the manner how the total magnetic flux through the coil changes. Hence it does not matter whether the magnet is drawn away from the coil (red light) or the coil is taken away from the magnet (also red light). Therefore the correct answer in the situation where the coil is lifted is red light. Fortunately, the post-discussion responses by the students showed a shift towards the correct answer (for the total count, the red-green opinions divided roughly evenly).

Some of the students' reflections for the flashing of red light:

Student C: "The direction of the current depends on which way the magnetic field changes. When the magnet is taken away from the coil, the field reduces. When the coil is taken away from the magnet, the field reduces."

Student D: "Considering the outcome, it is all the same, in which way the magnetic field is reduced."
Sometimes the justification of the answers was ambigious:

Student E: "In principle, the movement is the same, but reversed."

\section{Student F: "My friend had the same [answer]"}

In retrospect, also the administration of the test should have been given more careful attention. Considerable confusion could be noticed from some of the questionnaires:

Student G: "My answer was wrong, because I remembered the colors wrong."

Student H: "You could wait until everyone gets an answer sheet before the question is given!"

Note that the language of instruction was Finnish as well as the students' responses. The samples above have been translated in a way to maintain the meaning, contents, and style of the answer as accurately as possible.

\subsection{Confidence}

As to the confidence of the students, it seems that the majority were definitely sure or probably sure of their answers, and this confidence increased after the discussion: only $15 \%$ said that their answer is just a guess (pre-discussion), and the corresponding figure for post-discussion was $12 \%$. The number of students who were definitely sure that their answer was right increased considerably after the discussion: from $31 \%$ to $46 \%$. Of course, even after discussion there were students who were definitely convinced about the correctness of their wrong answer.

As a final note, the quantitative results in Table 1 and Figure 3 are averages over the two lectures given six years apart in time. The position of the Dynamic Field Theory course in the second-year schedule of the Electrical Engineering curriculum, as well as the teacher, have remained the same. Also the course material and the structure of the Faraday's law lecture has been very similar in these two 

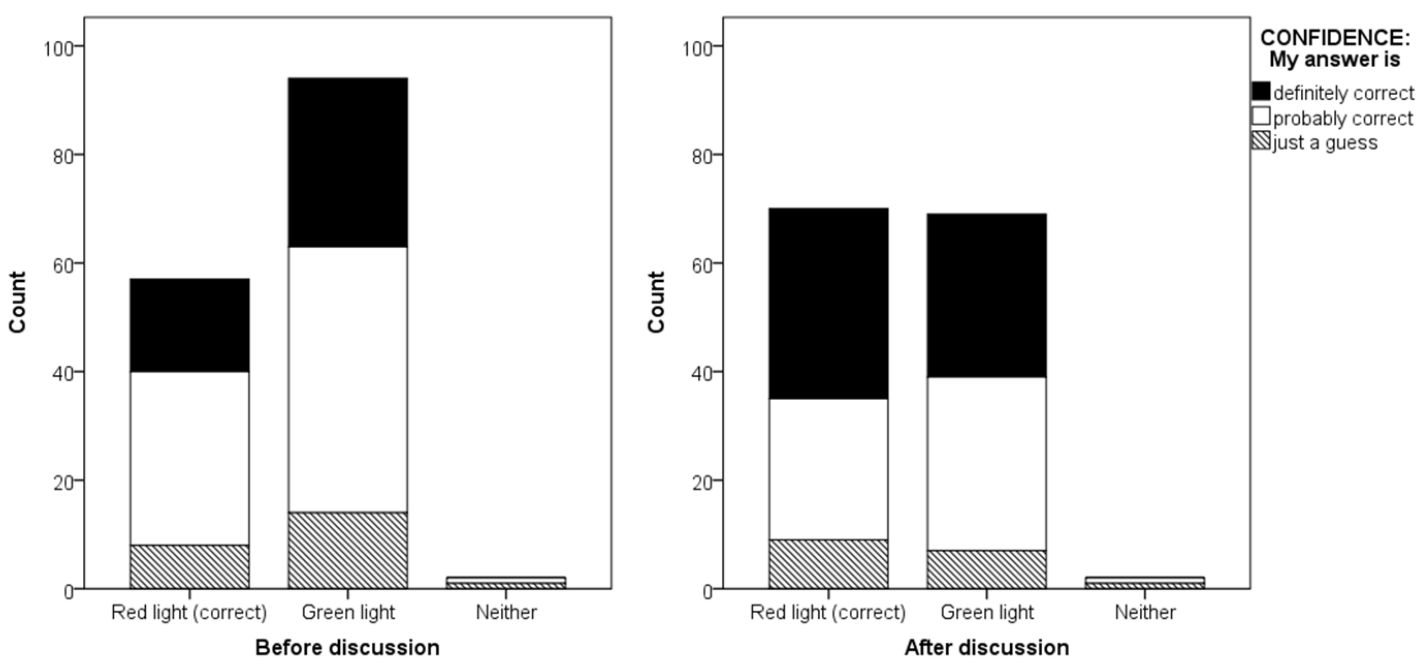

Figure 3: The distribution of students' answers and confidence levels before and after the discussion. The 2005 and 2011 data are combined. Data of those participants who gave no answer for the post-discussion questions were removed from the data set. The remaining sample size was 155 .

years. Of course, the individual students and hence the population have been different. Indeed, when the results were separated into the two classes, there were clear differences in the outcomes. The positive effect of the discussion in increasing the share of right answers was clearly larger in 2005 compared to 2011, as can be seen from Table 1 . This is, however, not necessarily a consequence of a decrease of students' capabilities. A more likely explanation is the nature of the coil-magnet problem itself. The relativity of the movement and temporal change of the absolute flux through the coil are two equally plausible logical ways of approaching the situation. These two different attractors are competing in a student's mind to determine her/his prediction for the correct color that will flash. In this kind of situation, the exact wording of the teacher during the preparation phase and the amount of time given for the students to make up their minds can have a strong influence on the general outcome of the responses.

\section{References}

[1] R. H. Romer, What do "voltmeters" measure? Faraday's law in multiply connected region, American Journal of Physics, vol. 50, no. 12, pp. 1089-1093, December 1982.

[2] J. A. Kong, Electromagnetic Wave Theory, Wiley, 1986.

[3] R. E. Collin, Field Theory of Guided Waves, Second Edition, IEEE Press, 1990.

[4] H. A. Haus, J. Melcher, R. Electromagnetic Fields and Energy, Prentice Hall, 1989.

[5] D. S. Jones, The Theory of Electromagnetism, Pergamon Press, 1964.
[6] D. K. Cheng, Field and Wave Electromagnetics, Addison Wesley, 1989.

[7] G. L. Pollack, D. R. Stump, Electromagnetism, Addison Wesley, 2002.

[8] J. D. Kraus, Electromagnetics, Fourth Edition, McGraw-Hill, 1992.

[9] J. D. Jackson, Classical Electrodynamics, Third Edition, John Wiley, 1999.

[10] B. M. Notaroš, Electromagnetics, Pearson, 2011.

[11] J. R. Reitz, F. J. Milford, R. W. Christy, Foundations of Electromagnetic Field Theory, Addison Wesley, 1993.

[12] Z. Popović, B. D. Popović, Introductory Electromagnetics, Prentice Hall, 1999.

[13] I. Lindell, A. Sihvola, Sähkömagneettinen kenttäteoria. I. Staattiset kentät, Otatieto, Helsinki, 1995.

[14] A. Sihvola, I. Lindell, Sähkömagneettinen kenttäteoria. I. Dynaamiset kentät, Otatieto, Helsinki, 1996.

[15] E. Mazur, Peer Instruction: A user's manual, Series in Educational Innovation, Prentice Hall, Upper Saddle River, NJ, 1997. 